\section{Fatalities from Brucellosis}

SIR,-The results of a study of brucellosis fatalities in the British Isles may be of topical interest in view. of the small current attention being given to the elimination of this readily preventable disease-to which you referred in a leading article recently (January 26, p. 204).

1 to $2 \%$ of recognized cases of brucellosis are fatal, and 45 fatal cases have previously been described in the literature from the British Isles.

Under the aegis of the Agricultural Research Council and with the help of the Registrar-General or equivalents and the co-operation of hospital consultants, a series of 27 brucellosis deaths occurring in the British Isles mainly between 1950 and 1960 (1 in 1949) has been studied. Case reports were available in 15 of these cases.

The terminal conditions in these 27 cases were: hepatic 9, cardiovascular 9 (including 2 with pulmonary embolus, in males), other conditions 9 (among which were 2 with thrombocytopenia and 1 obscure neurological state). The age distribution of these cases was: 1-10 years, two ; 21-30, one ; $31-40$, four ; 41-50, three ; 51-60, seven ; over 60 , eight ; age unknown, two.

Among the 15 cases where hospital notes were available, eight cases appeared to be in an advanced stage, four were fairly recent, and three were of indeterminate duration. These cases were treated with various antibiotics in different combinations without effect. Blood culture was positive in two cases in life and in another on post-mortem specimen. Agglutination titres ranged from $1 / 160$ to $1 / 16,000$. In five of the 15 cases fever was the predominant symptom, three presented with general malaise, and one with abdominal pain and diarrhoea; one had "infective hepatitis," one a rash with bronchitis, and one had breathlessness with swollen ankles. The type of fever was irregular in six, remittent in three, and in five had an undulant characteristic. Associated with brucellosis were diverticulitis in one case, diabetes in another, and diabetes with haemochromatosis in a third. In three cases granulomata were found in the liver, two at post-mortem and one from laparotomy biopsy. One of these had granulomata in other organs and an Aschoff-like body in the myocardium.

There was no evidence of investigation of cases in regard to sources of infection.

Of 56 fatalities reported in Britain where epidemiological data were available $26.8 \%$ occurred in persons occupationally connected with farming, dairying, or veterinary services; the male/female ratio was $2: 1$.

There is a need for earlier and more definite methods of diagnosis, more information about the disease histologically (liver puncture was not used diagnostically), more post-mortem material, and more knowledge about the dosage and combination of antibiotics in brucellosis.

Official notification of cases and fatalities would ensure follow-up studies of sources of infection, which in these cases had not been attempted.-I am, etc.,

$$
\text { Department of Public Health, P. W. BothWELl. }
$$
Bristol 2.

\section{Chloramphenicol and Blood Dyscrasias}

SIR,--May I congratulate Mr. W. D. Park on his objective letter (April 6, p. 950) and also say how delighted I was to find there is at least one other medical practitioner who realizes the clinical value of chloramphenicol (Dr. G. Riddell Royston, April 27, p. 1161).
As Dr. Riddell Royston has done, I have used this antibiotic as the initial treatment of acute exacerbations of upper respiratory infection for the past five and a half years in general practice. According to my records the rate of prescription has varied between 32 and 114 individual courses per month and I calculate that during the whole period I have prescribed something in excess of five thousand courses of treatment.

Each course of treatment has consisted of $250 \mathrm{mg}$. q.d.s. for four to six days. This is more or less the same regime as that adopted by Dr. Riddell Royston, but I can go a stage further than he, for he states that a second course is never given. I have on many occasions given a second course, provided clinical improvement has occurred with the first course, and I have prescribed up to six courses in one year for people with chronic bronchitis.

During the five-and-a-half-year period I have had one death from leukaemia, and this person had never, to my knowledge, taken chloramphenicol. Three people have had severe skin reactions. One was an eczematous reaction in a middle-aged man with a previous history of eczema. One was a vesicular eruption on the trunk and limb extremities. The other was an erythema nodosum involving all limb extremities. All responded very rapidly on withdrawal of the chloramphenicol and administration of an antihistamine. The severe eczematous reaction occurred in a man who had had a mild reaction to chloramphenicol two years previously, but unfortunately I failed to note this when prescribing the second course.

Fourteen people have complained of mild reactions, mainly of an erythematous nature, and these have occurred as early as the first day and as late as the fifth day of a course.-I am, etc.,

Birmingham 6.

Gilbert R. Smith.

\section{Quality Control of Drugs}

SIR,-I would like to reply to Mr. Frank G. Stock's letter (April 13, p. 1024).

First I would like to make it clear that the batch of penicillin tablets purchased by this Board was acquired from a different firm and was of different manufacture to those referred to by Mr. Stock in his address as having been supplied to retail chemists at about the same time.

Secondly, an analyst's report accompanying the tablets, and an independent analyst's report obtained by the Board, showed that the batch was within the B.P. limit. A second assay carried out by the manufacturers before any communication was received from Mr. Stock again confirmed that the tablets were satisfactory. When Mr. Stock later suggested that the batch was below the limit, samples were sent to the Government laboratory for further tests. The first report from the Government laboratory covering 11 assays indicated that the batch was within the B.P. limit. A subsequent test carried out by the Government laboratory was not entirely conclusive, as one technician produced a result indicating that the batch was well within the B.P. limit and another technician a result indicating that the batch was below the limit.

In view of the results of the original tests and the first results obtained by the Government laboratory, which were all at variance with Mr. Stock's findings, the Board did not consider that the batch was suspect enough to warrant its withdrawal. By the time the Government 